\title{
Temperature Sensation in Parkinson's Disease Measured by Quantitative Sensory Testing
}

Michaela Kaiserova ( $\nabla$ michaela.kaiserova@fnol.cz)

Palacky University Olomouc Faculty of Medicine and Dentistry: Univerzita Palackeho v Olomouci Lekarska fakulta https://orcid.org/0000-0003-4776-9055

Zuzana Grambalova

Palacky University Olomouc Faculty of Medicine and Dentistry: Univerzita Palackeho v Olomouci Lekarska fakulta

Pavel Otruba

Palacky University Olomouc Faculty of Medicine and Dentistry: Univerzita Palackeho v Olomouci Lekarska fakulta Anetta Janova

Palacky University Olomouc Faculty of Medicine and Dentistry: Univerzita Palackeho v Olomouci Lekarska fakulta Jana Zapletalova

Palacky University Olomouc Faculty of Medicine and Dentistry: Univerzita Palackeho v Olomouci Lekarska fakulta

Katerina Mensikova

Palacky University Olomouc Faculty of Medicine and Dentistry: Univerzita Palackeho v Olomouci Lekarska fakulta

Petr Kanovsky

Palacky University Olomouc Faculty of Medicine and Dentistry: Univerzita Palackeho v Olomouci Lekarska fakulta

\section{Research}

Keywords: Parkinson's disease, Quantitative sensory testing, Cold detection threshold, Warm detection threshold

Posted Date: November 13th, 2020

DOI: https://doi.org/10.21203/rs.3.rs-104776/v1

License: (c) (i) This work is licensed under a Creative Commons Attribution 4.0 International License. Read Full License 


\section{Abstract}

\section{Background}

The pathophysiology of abnormal temperature sensation in Parkinson's disease (PD) remains unclear. Abnormal thermal detection does not seem to depend on the dopaminergic deficit, suggesting that other systems play a role in these changes, probably both central and peripheral.

Methods

We measured thermal detection thresholds (TDT) using quantitative sensory testing (QST) in 28 patients with PD and compared them with 15 healthy controls.

Results

Of 28 patients, $21 \%$ had increased TDT according to the normative data. TDT were higher on the dominant side. No correlation between TDT and disease duration, severity of motor impairment, and dopaminergic therapy was observed. $50 \%$ of the patients had difficulty differentiating between warm and cold stimuli, as TDT were within the normal range in most of these patients.

Conclusions

These results suggest that abnormal thermal detection may be present from early stages of the disease and is more pronounced on the dominant side. Abnormal differentiation between the thermal stimuli suggest impaired central processing of thermal information.

\section{Introduction}

Apart from motor symptoms, Parkinson's disease (PD) is characterized by a number of non-motor features, such as autonomic dysfunction, sleep disturbances, sensory, neuropsychiatric, and gastrointestinal symptoms. Sensory symptoms are common in PD, usually manifested as pain, numbness, paresthesias, or dysesthesias. Patients often describe coldness and tingling or tightening of the muscles without objective evidence of sensory loss [1-3]. There have been several studies concerning peripheral nerve pathology in PD, such as polyneuropathy $[4,5]$, small fibre neuropathy $[6,7]$ or motor neuron disease $[8,9]$; some sensory symptoms are supposed to be caused by central pathology [10].

Only a limited number of studies have investigated thermal abnormalities in PD [11-19] and the underlying pathophysiology still remains unclear. Most of the published studies found increased thermal detection thresholds but no responsiveness to levodopa, suggesting that thermal abnormalities do not depend on dopaminergic deficit. Some studies found reduced thermal detection thresholds after STN-DBS (Deep Bran Stimulation of the Subthalamic Nucleus) [3,11, 14, 16, 20, 21], suggesting that central pathology plays a role in abnormal thermal sensation. Peripheral deafferentation probably also plays a role [15, 17], as small fiber neuropathy has been confirmed on skin biopsies in PD patients [6, 7].

The aim of our study was to describe the characteristics of temperature detection in PD. We measured thermal detection thresholds in PD using quantitative sensory testing (QST) and correlated the results with the disease duration, severity of motor impairment, and dosage of dopaminergic medication. We also assessed the side-to-side difference in thermal detection thresholds.

\section{Material And Methods}

The study protocol, was approved by the Ethics Committee of Palacky University in Olomouc; all participants gave their informed consent prior to their recruitment into the study. All procedures were performed in accordance with relevant guidelines and regulations.

Page 2/11 


\section{Patients and Controls}

The study included 28 patients with PD (11 male, 17 female; mean age 57.5 years, SD $=7.8)($ Table 1$)$ and 15 healthy agematched controls (mean age 58.2, SD = 10.2). The diagnosis of PD was established according to the UK Parkinson's Disease Society Brain Bank clinical diagnostic criteria [22]. None of the patients had dementia (25 patients underwent complete neuropsychological testing; 3 patients were administered the Mini-Mental State Examination (MMSE) with results within the normal range. All patients and controls underwent QST and a nerve conduction study (NSC). Of the 28 examined patients, 17 patients were treated with levodopa, dopamine agonist, or both; the other 11 were dopa naïve. 
Table 1

Patients'characteristics

\begin{tabular}{|c|c|c|c|c|c|c|c|c|c|}
\hline Patient & Sex & $\begin{array}{l}\text { Age } \\
\text { (years) }\end{array}$ & $\begin{array}{l}\text { Dominant } \\
\text { side }\end{array}$ & $\begin{array}{l}\text { Duration } \\
\text { of PD } \\
\text { (years) }\end{array}$ & H\&Y & $\begin{array}{l}\text { Abnormal } \\
\text { absolute } \\
\text { QST } \\
\text { values (1) }\end{array}$ & $\begin{array}{l}\text { Discrimination } \\
\text { abnoramlities }\end{array}$ & $\begin{array}{l}\text { Antiparkinsonian } \\
\text { drugs }\end{array}$ & $\begin{array}{l}\text { Levodopa } \\
\text { equivalent } \\
\text { (mg) (2) }\end{array}$ \\
\hline 1 & $M$ & 54 & $\mathrm{~L}$ & 1 & 2,5 & & & & \\
\hline 2 & $\mathrm{~F}$ & 52 & $\mathrm{~L}$ & 6 & 3 & $\begin{array}{l}\text { cold } \\
\text { dominant }\end{array}$ & & LD, rop & 880 \\
\hline 3 & M & 72 & $\mathrm{~L}$ & 3 & 3 & & & LD, pram & 360 \\
\hline 4 & $\mathrm{~F}$ & 61 & $\mathrm{~L}$ & 1 & 1 & & & & \\
\hline 5 & M & 63 & $\mathrm{~L}$ & 1 & 2 & $\begin{array}{l}\text { cold } \\
\text { dominant }\end{array}$ & bilateral & & \\
\hline 6 & $M$ & 59 & $\mathrm{~L}$ & 5 & 2 & & bilateral & LD, pram & 1010 \\
\hline 7 & $\mathrm{~F}$ & 61 & $\mathrm{~L}$ & 7 & 2 & & & LD, rop & 980 \\
\hline 8 & $\mathrm{~F}$ & 62 & $\mathrm{~L}$ & 2 & 1 & & & & \\
\hline 9 & $\mathrm{~F}$ & 59 & $\mathrm{~L}$ & 11 & 2 & & & LD, rop & 720 \\
\hline 10 & $\mathrm{~F}$ & 47 & $\mathrm{~L}$ & 1 & 1 & & bilateral & & \\
\hline 11 & $\mathrm{~F}$ & 56 & $\mathrm{~L}$ & 3 & 1 & & bilateral & rop & 320 \\
\hline 12 & $\mathrm{~F}$ & 59 & $\mathrm{~L}$ & 1 & 1 & $\begin{array}{l}\text { warm } \\
\text { dominant }\end{array}$ & Dominant side & & \\
\hline 13 & $\mathrm{~F}$ & 64 & $\mathrm{~L}$ & 2 & 2 & & & & \\
\hline 14 & $M$ & 54 & $\mathrm{~L}$ & 2 & 1 & & bilateral & & \\
\hline 15 & $M$ & 58 & $\mathrm{R}$ & 3 & 2 & & bilateral & LD, rot & 880 \\
\hline 16 & Z & 55 & $\mathrm{R}$ & 3 & 1 & & & LD, rop & 660 \\
\hline 17 & M & 56 & $\mathrm{R}$ & 2 & 2 & & & LD & 1000 \\
\hline 18 & Z & 61 & $\mathrm{R}$ & 1 & 2 & & bilateral & & \\
\hline 19 & $M$ & 56 & $\mathrm{R}$ & 1 & 1 & & & pram & 210 \\
\hline 20 & Z & 50 & $\mathrm{R}$ & 1 & 1 & & Dominant side & & \\
\hline 21 & Z & 46 & $\mathrm{R}$ & 0,5 & 1 & & & & \\
\hline 22 & Z & 72 & $\mathrm{R}$ & 11 & 3 & & & LD, rop & 1230 \\
\hline 23 & Z & 52 & $\mathrm{R}$ & 6 & 2 & & bilateral & LD & 1000 \\
\hline 24 & $M$ & 37 & $\mathrm{R}$ & 10 & 2 & & Dominant side & LD, rop & 880 \\
\hline
\end{tabular}

1) Absolute QST values according to the normative data (Rolke et al., 2006)

2) Levodopa equivalent (Moller et al., 2005)

Moller, J.C., et al., 2005. Pharmacotherapy of Parkinson's disease in Germany. J Neurol. 252, 926 - 35.

Rolke, R., et al., 2006. Quantitative sensory testing in the German Research Network on Neuropathic Pain (DFNS): standardized protocol and reference values. Pain. 123, $231-43$. 


\begin{tabular}{|c|c|c|c|c|c|c|c|c|c|}
\hline Patient & Sex & $\begin{array}{l}\text { Age } \\
\text { (years) }\end{array}$ & $\begin{array}{l}\text { Dominant } \\
\text { side }\end{array}$ & $\begin{array}{l}\text { Duration } \\
\text { of PD } \\
\text { (years) }\end{array}$ & H\&Y & $\begin{array}{l}\text { Abnormal } \\
\text { absolute } \\
\text { QST } \\
\text { values (1) }\end{array}$ & $\begin{array}{l}\text { Discrimination } \\
\text { abnoramlities }\end{array}$ & $\begin{array}{l}\text { Antiparkinsonian } \\
\text { drugs }\end{array}$ & $\begin{array}{l}\text { Levodopa } \\
\text { equivalent } \\
\text { (mg) (2) }\end{array}$ \\
\hline 25 & $M$ & 60 & $\mathrm{R}$ & 1 & 1 & $\begin{array}{l}\text { warm } \\
\text { non- } \\
\text { dominant }\end{array}$ & & rop & 320 \\
\hline 26 & Z & 55 & $\mathrm{R}$ & 9 & 2 & $\begin{array}{l}\text { cold } \\
\text { dominant, } \\
\text { warm } \\
\text { bilateraly }\end{array}$ & bilateral & pram & 210 \\
\hline 27 & Z & 73 & $\mathrm{R}$ & 11 & 4 & $\begin{array}{l}\text { warm } \\
\text { bilateraly }\end{array}$ & bilateral & LD & 400 \\
\hline 28 & $M$ & 57 & $\mathrm{R}$ & 8 & 3 & & $\begin{array}{l}\text { non-dominant } \\
\text { side }\end{array}$ & LD, rop & 720 \\
\hline
\end{tabular}

1) Absolute QST values according to the normative data (Rolke et al., 2006)

2) Levodopa equivalent (Moller et al., 2005)

Moller, J.C., et al., 2005. Pharmacotherapy of Parkinson's disease in Germany. J Neurol. 252, 926 - 35.

Rolke, R., et al., 2006. Quantitative sensory testing in the German Research Network on Neuropathic Pain (DFNS): standardized protocol and reference values. Pain. 123, $231-43$.

\section{Quantitative sensory testing}

Thermal thresholds (cold detection threshold, CDT, and warm detection threshold, WDT) were tested using Medoc,TSA 2001, Israel. Testing was performed on the dorsum of both feet using the methods of limits [23]. Five stimuli of each modality were delivered. In the next step, 10 thermal stimuli were applied in random order and the patient was asked to identify the type of the stimulus (warm vs. cold).

\section{Nerve conduction study}

NCS was performed using the Keypoint IV® system (Medtronic, Tonstakken, Denmark) under standardized conditions [24]. The motor conduction of the deep peroneal and tibial nerves and sensory conduction of the sural and superficial peroneal nerves were measured.

\section{Statistical analysis}

Statistical analysis was performed using IBM SPSS Statistics for Windows, Version 22.0 (Armonk, NY: IBM Corp.). The Wilcoxon signed-ranks test was used to evaluate paired measurements (side-to side differences in thermal detection thresholds in patients and controls, comparison of thermal detection thresholds on dominant vs. non-dominant side). The Mann-Whitney $U$ test was used to compare differences between patients and controls. The relationships between thermal detection thresholds and selected quantitative parameters (disease duration, Hoehn \& Yahr score) were assessed using the Spearman's correlation. Tests were performed at the significance level of 0.05 .

\section{Results}

\section{Subjects}

Patients' characteristics are listed in Table 1. The mean disease duration of PD patients was 4.1 years (minimum 0.5 years, maximum 11 years), SD 3.6. Disease duration was measured from the time when the first motor symptoms occurred. Hoehn \& Yahr score ranged from I to III. 


\section{Nerve conduction studies and Quantitative sensory testing}

Conduction studies excluded polyneuropathy in all subjects, both patients and controls. WDT and CDT in PD and controls are listed in Tables 2 and 3. In the control group, the mean temperature was obtained from the average values of both feet; the mean CDT was $28.9^{\circ} \mathrm{C}$ (median $29.1^{\circ} \mathrm{C}$ ), the mean WDT was 38.3 (median $37.6^{\circ} \mathrm{C}$ ). Six PD patients $(21 \%)$ had increased thermal detection thresholds (CDT or WDT or both) according to the normative data given by Rolke et al. (2006) [25]. All control subjects had thermal thresholds within the normal range. PD patients showed significantly higher thermal detection thresholds on the more affected side than on the non-dominant side (cold $P=0.015$, warm $P=0.045$ ) (Table 2, Figs. 1 and 2). In the control group, there was no side-to-side difference in the thermal detection thresholds (cold $P=0.099$, warm $P=0.615)(T a b l e ~ 3)$.

Fourteen patients $(50 \%)$ had difficulties with differentiating between warm and cold stimuli. No significant correlation between thermal detection thresholds and disease duration, Hoehn \& Yahr score, or dopaminergic drug intake was observed.

Table 2

Cold and warm detection thresholds in Parkinson's disease patients

\begin{tabular}{|c|c|c|c|c|c|c|}
\hline & $\begin{array}{l}\text { Mean } \\
\left({ }^{\circ} \mathrm{C}\right)\end{array}$ & SD & $\begin{array}{l}\text { Median } \\
\left({ }^{\circ} \mathrm{C}\right)\end{array}$ & $\begin{array}{l}\text { Minimum } \\
\left({ }^{\circ} \mathrm{C}\right)\end{array}$ & $\begin{array}{l}\text { Maximum } \\
\left({ }^{\circ} \mathrm{C}\right)\end{array}$ & Wilcoxon signed-ranks test $(P)$ \\
\hline dominant CDT & 27,3 & 2,6 & 27,9 & 18,2 & 30,2 & \multirow[t]{2}{*}{0,015} \\
\hline non-dominant CDT & 28,0 & 2,5 & 28,8 & 19,0 & 31,0 & \\
\hline dominant WDT & 39,9 & 3,5 & 38,9 & 34,6 & 47,4 & \multirow[t]{2}{*}{0,045} \\
\hline $\begin{array}{l}\text { non-dominant } \\
\text { WDT }\end{array}$ & 39,2 & 3,7 & 38,6 & 35,0 & 48,9 & \\
\hline
\end{tabular}

Table 3

Cold and warm detection thresholds in control group

\begin{tabular}{|c|c|c|c|c|c|c|}
\hline & $\begin{array}{l}\text { Mean } \\
\left({ }^{\circ} \mathrm{C}\right)\end{array}$ & SD & $\begin{array}{l}\text { Median } \\
\left({ }^{\circ} \mathrm{C}\right)\end{array}$ & $\begin{array}{l}\text { Minimum } \\
\left({ }^{\circ} \mathrm{C}\right)\end{array}$ & $\begin{array}{l}\text { Maximum } \\
\left({ }^{\circ} \mathrm{C}\right)\end{array}$ & Wilcoxon signed-ranks test $(P)$ \\
\hline Left foot - CDT & 29,1 & 2,0 & 29,6 & 23,2 & 31,0 & \multirow[t]{2}{*}{0,099} \\
\hline Right foot - CDT & 28,6 & 2,3 & 28,9 & 21,2 & 31,1 & \\
\hline Left foot - WDT & 38,4 & 2,9 & 37,6 & 34,3 & 43,0 & \multirow[t]{2}{*}{0,615} \\
\hline Right foot - WDT & 38,1 & 2,9 & 37,6 & 34,4 & 45,2 & \\
\hline
\end{tabular}

\section{Discussion}

Little is known about the abnormal temperature sensation in PD, although the impaired temperature sensation may negatively affect the quality of life in PD. Using QST as a diagnostic method, several studies found an increased temperature threshold in PD [14, 16-18]; other studies did not find any difference between the patients and the control group [13, 26]. In our study, only $21 \%$ of the patients had increased thermal detection thresholds (CDT or WDT or both) according to the normative data given by Rolke et al [25]. With regard to these findings, abnormal thermal thresholds measured by QST are present in the minority of patients and do not seem to be a typical finding in PD. The study of Lin et al. found similar results [15]. In their cohort of PD patients, $32.1 \%$ had abnormal thermal thresholds. 
On the other hand, $50 \%$ of the patients had difficulty differentiating between warm and cold stimuli; increased thermal detection thresholds, however, were present only in 4 of those patients $(28 \%)$. This means that patients may have preserved ability to detect a thermal stimulus per se, demonstrated as a normal thermal threshold in QST, but they have difficulty differentiating the type of the applied stimulus. This suggests a deficit in the central processing of the thermal information.

Previous studies $[14,17]$ found no difference in somatosensory thresholds between the dominant and non-dominant side. Our observation was different. PD patients in our cohort showed higher thermal thresholds on the more affected side compared to the non-dominant side, both for cold and warm stimuli.

Next, we focused on the disease duration, severity of the disease, and antiparkinsonian drug intake as possible modifying parameters of temperature detection thresholds. There was no correlation of CDT or WDT with the disease duration, suggesting that abnormal thermal sensation may be present from the early stages of PD. This finding is consistent with the reports by Nolano et al. $[17,18]$ and supported by the study of Strobel et al., who found abnormal thermal thresholds in idiopathic REM sleep behavior disorder [27], which is considered a frequent and important premotor symptom of PD [18].

The severity of motor impairment, as measured by Hoehn \& Yahr scores, did not correlate with the thermal thresholds, suggesting no association between abnormal thermal detection thresholds and dopaminergic deficit. This is also supported by the finding of no significant difference in WDT or CDT in PD patients who take levodopa and who are dopa naïve. Our findings are consistent with other studies that showed no effect of dopamine treatment on thermal sensation $[13,14]$.

\section{Conclusions}

Abnormal thermal detection may be present from the early stages of PD and is more pronounced on the more affected side. But only a minority of patients have increased thermal detection thresholds as measured by QST. More commonly observed is difficulty differentiating between warm and cold stimuli, which suggests an impaired central processing of thermal information.

\section{List Of Abbreviations}

PD Parkinson's disease

STN-DBS Deep Bran Stimulation of the Subthalamic Nucleus

QST Quantitative sensory testing

NCS Nerve conduction study

CDT Cold detection threshold

WDT Warm detection threshold

MMSE Mini-Mental State Examination

\section{Declarations}

\section{Ethics approval and consent to participate}

The study protocol, was approved by the Ethics Committee of Palacky University in Olomouc; all participants gave their informed consent prior to their recruitment into the study. All procedures were performed in accordance with relevant guidelines and regulations.

\section{Consent for publication}

All contributing authors have given their consent for the publication of this study.

Page $7 / 11$ 
Availability of data and material

The data that support the findings of this study are available from the corresponding author, upon reasonable request.

\section{Competing interests}

The authors declare that they have no conflicts of interest.

\section{Funding}

This study was supported by the European Regional Development Fund - Project ENOCH (No.

Z.02.1.01/0.0/0.0/16_019/0000868); by the grant project of the Ministry of Health of the Czech Republic for the conceptual development of a research organization (FNOL, 0098892) - RVO FNOL 2020.

\section{Author contributions}

M.K. study design, patient examination, data collection and analysis, drafting the initial manuscript. Z.G., P.O., A.J. patient examination, data collection. J.Z. statistical analysis. PK study design, study supervision, review of the final version of the manuskript

\section{Acknowledgments}

Not applicable.

\section{References}

1. Bayulkem, K. and G. Lopez, Clinical approach to nonmotor sensory fluctuations in Parkinson's disease. J Neurol Sci, 2011. 310(1-2): p. 82-5.

2. Snider, S.R., et al., Primary sensory symptoms in parkinsonism. Neurology, 1976. 26(5): p. 423-9.

3. Cury, R.G., et al., Sensory abnormalities and pain in Parkinson disease and its modulation by treatment of motor symptoms. Eur J Pain, 2016. 20(2): p. 151-65.

4. Zis, P., et al., Peripheral neuropathy in idiopathic Parkinson's disease: A systematic review. J Neurol Sci, 2017. 378: p. 204209.

5. Grambalova, Z., et al., Peripheral neuropathy in Parkinson's disease. Neuro Endocrinol Lett, 2015. 36(4): p. 363-7.

6. Jeziorska, M., et al., Small Fibre Neuropathy in Parkinson's Disease: Comparison of Skin Biopsies from the More Affected and Less Affected Sides. Journal of Parkinsons Disease, 2019. 9(4): p. 761-765.

7. Ghasemi, M. and Y.A. Rajabally, Small fiber neuropathy in unexpected clinical settings: a review. Muscle Nerve, 2020.

8. Uitti, R.J., et al., Neurodegenerative 'overlap' syndrome: Clinical and pathological features of Parkinson's disease, motor neuron disease, and Alzheimer's disease. Parkinsonism Relat Disord, 1995. 1(1): p. 21-34.

9. Farnikova, K., et al., Coexistence of parkinsonism, dementia and upper motor neuron syndrome in four Czech patients. J Neurol Sci, 2010. 296(1-2): p. 47-54.

10. Zhu, M., et al., Sensory symptoms in Parkinson's disease: Clinical features, pathophysiology, and treatment. J Neurosci Res, 2016. 94(8): p. 685-92.

11. Ciampi de Andrade, D., et al., Subthalamic deep brain stimulation modulates small fiber-dependent sensory thresholds in Parkinson's disease. Pain, 2012. 153(5): p. 1107-13.

12. Conte, A., et al., Pathophysiology of somatosensory abnormalities in Parkinson disease. Nat Rev Neurol, 2013. 9(12): p. 687-97.

13. Djaldetti, R., et al., Quantitative measurement of pain sensation in patients with Parkinson disease. Neurology, 2004. 62(12): p. 2171-5. 
14. Gierthmuhlen, J., et al., Influence of deep brain stimulation and levodopa on sensory signs in Parkinson's disease. Mov Disord, 2010. 25(9): p. 1195-202.

15. Lin, C.H., et al., Pathophysiology of Small-Fiber Sensory System in Parkinson's Disease: Skin Innervation and Contact Heat Evoked Potential. Medicine (Baltimore), 2016. 95(10): p. e3058.

16. Maruo, T., et al., Deep brain stimulation of the subthalamic nucleus improves temperature sensation in patients with Parkinson's disease. Pain, 2011. 152(4): p. 860-5.

17. Nolano, M., et al., Sensory deficit in Parkinsons disease: evidence of a cutaneous denervation. Brain, 2008. 131: p. 19031911.

18. Nolano, M., et al., Loss of cutaneous large and small fibers in naive and L-dopa-treated PD patients. Neurology, 2017. 89(8): p. 776-784.

19. Spielberger, S., et al., The influence of deep brain stimulation on pain perception in Parkinson's disease. Mov Disord, 2011. 26(7): p. 1367-8; author reply 1368-9.

20. Belasen, A., et al., Effect of low-frequency deep brain stimulation on sensory thresholds in Parkinson's disease. J Neurosurg, 2017. 126(2): p. 397-403.

21. Cury, R.G., et al., Subthalamic deep brain stimulation modulates conscious perception of sensory function in Parkinson's disease. Pain, 2016. 157(12): p. 2758-2765.

22. Gibb, W.R. and A.J. Lees, The relevance of the Lewy body to the pathogenesis of idiopathic Parkinson's disease. J Neurol Neurosurg Psychiatry, 1988. 51(6): p. 745-52.

23. Chong, P.S. and D.P. Cros, Technology literature review: quantitative sensory testing. Muscle Nerve, 2004. 29(5): p. 734-47.

24. Stalberg, E., et al., Standards for quantification of EMG and neurography. Clin Neurophysiol, 2019. 130(9): p. 1688-1729.

25. Rolke, R., et al., Quantitative sensory testing in the German Research Network on Neuropathic Pain (DFNS): standardized protocol and reference values. Pain, 2006. 123(3): p. 231-43.

26. Frundt, O., et al., Quantitative Sensory Testing (QST) in Drug-Naive Patients with Parkinson's Disease. Journal of Parkinsons Disease, 2019. 9(2): p. 369-378.

27. Strobel, A.V., et al., Somatosensory function is impaired in patients with idiopathic REM sleep behaviour disorder. Sleep Medicine, 2018. 42: p. 83-89.

\section{Figures}




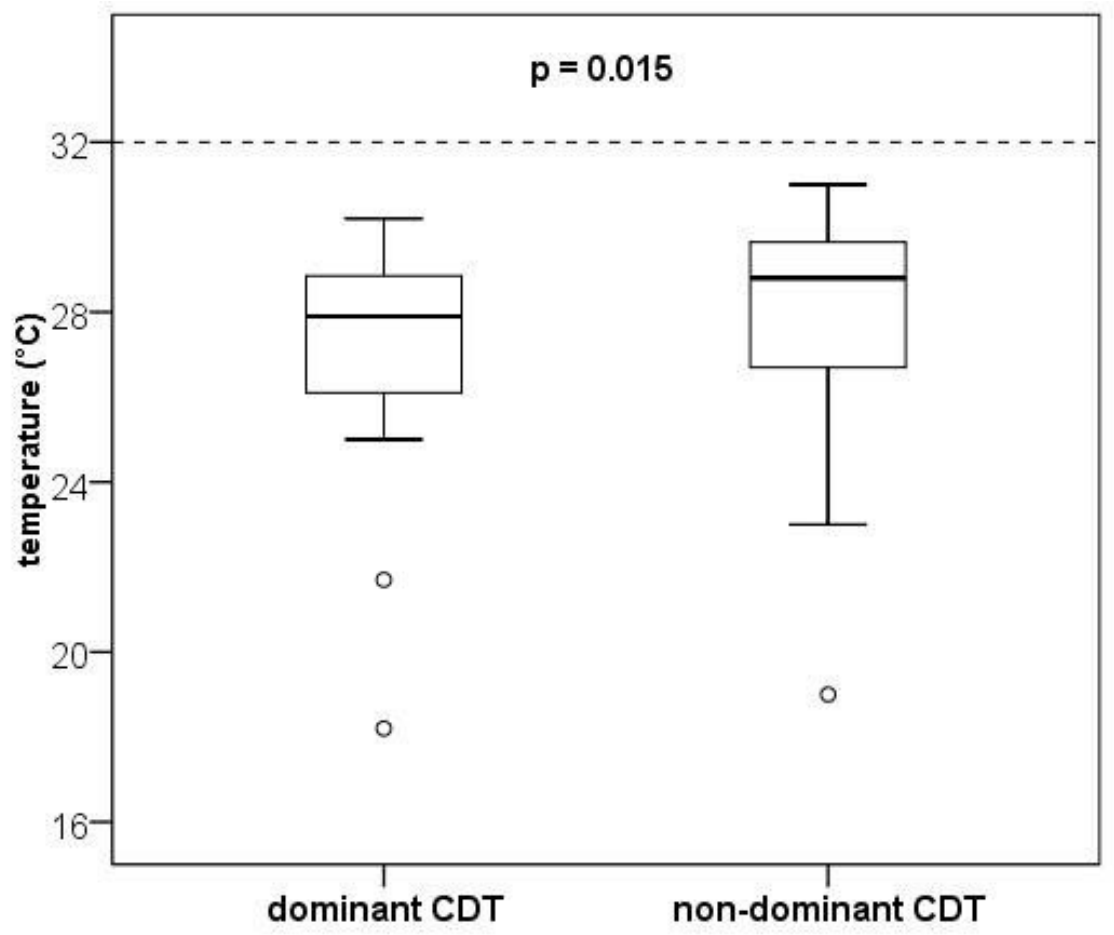

Figure 1

Cold detection threshold in Parkinson's disease patients. CDT, cold detection threshold; dominant, more affected side; nondominant, less affected side

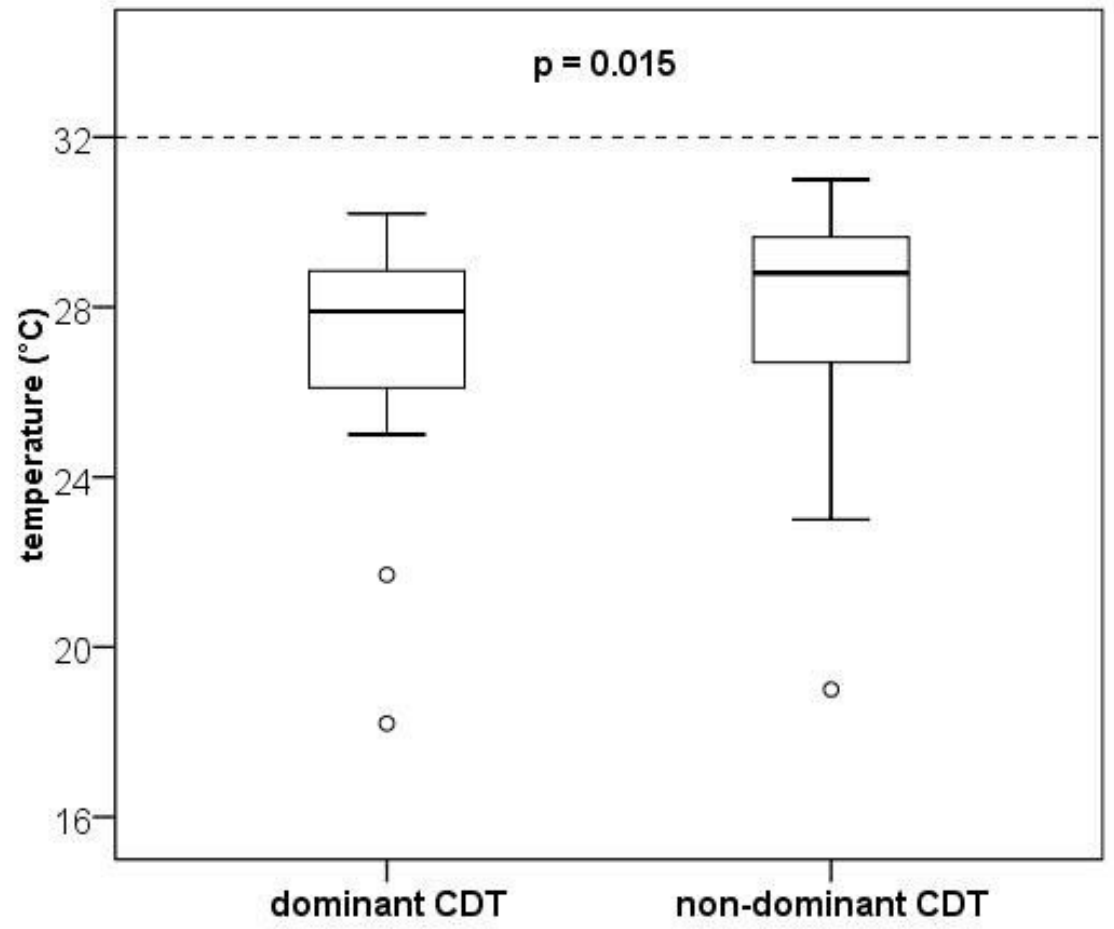

Figure 1

Cold detection threshold in Parkinson's disease patients. CDT, cold detection threshold; dominant, more affected side; nondominant, less affected side 


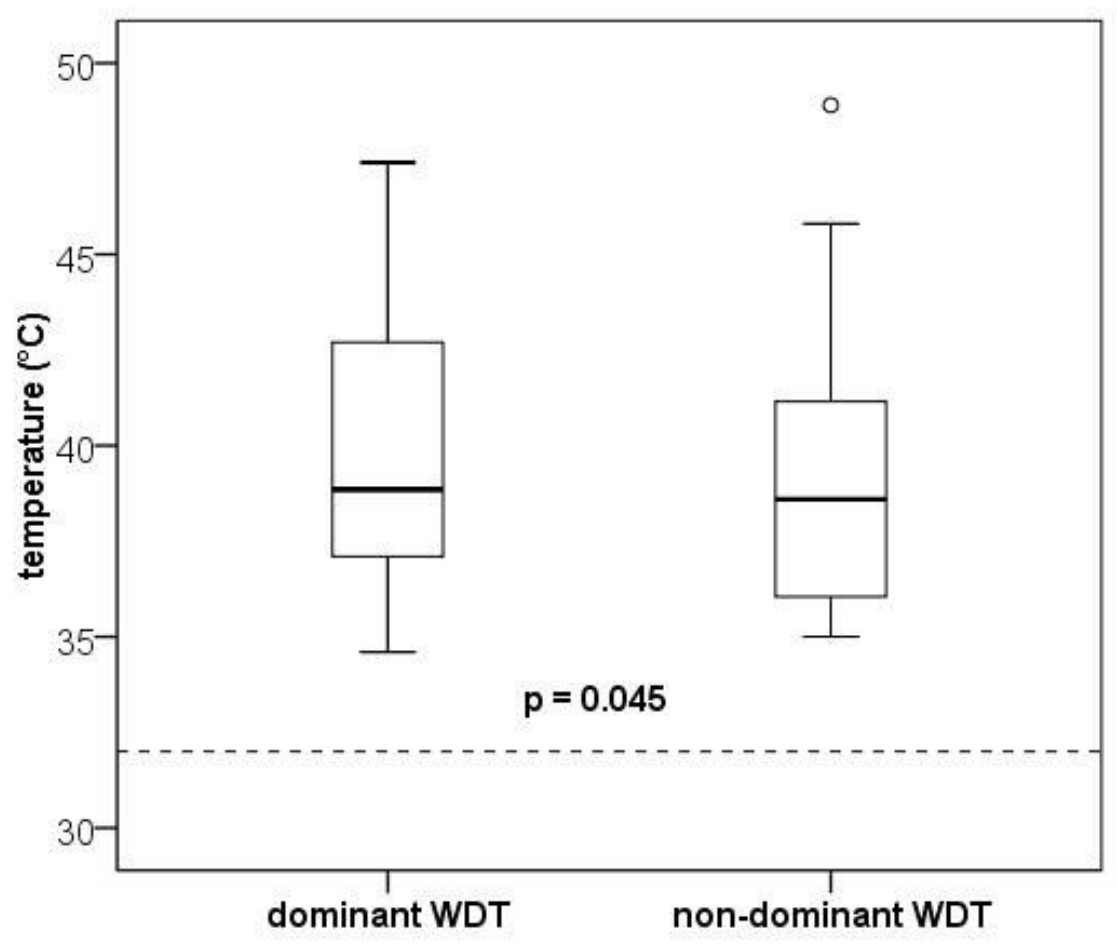

Figure 2

Warm detection threshold in Parkinson's disease patients. WDT, warm detection threshold; dominant, more affected side; nondominant, less affected side

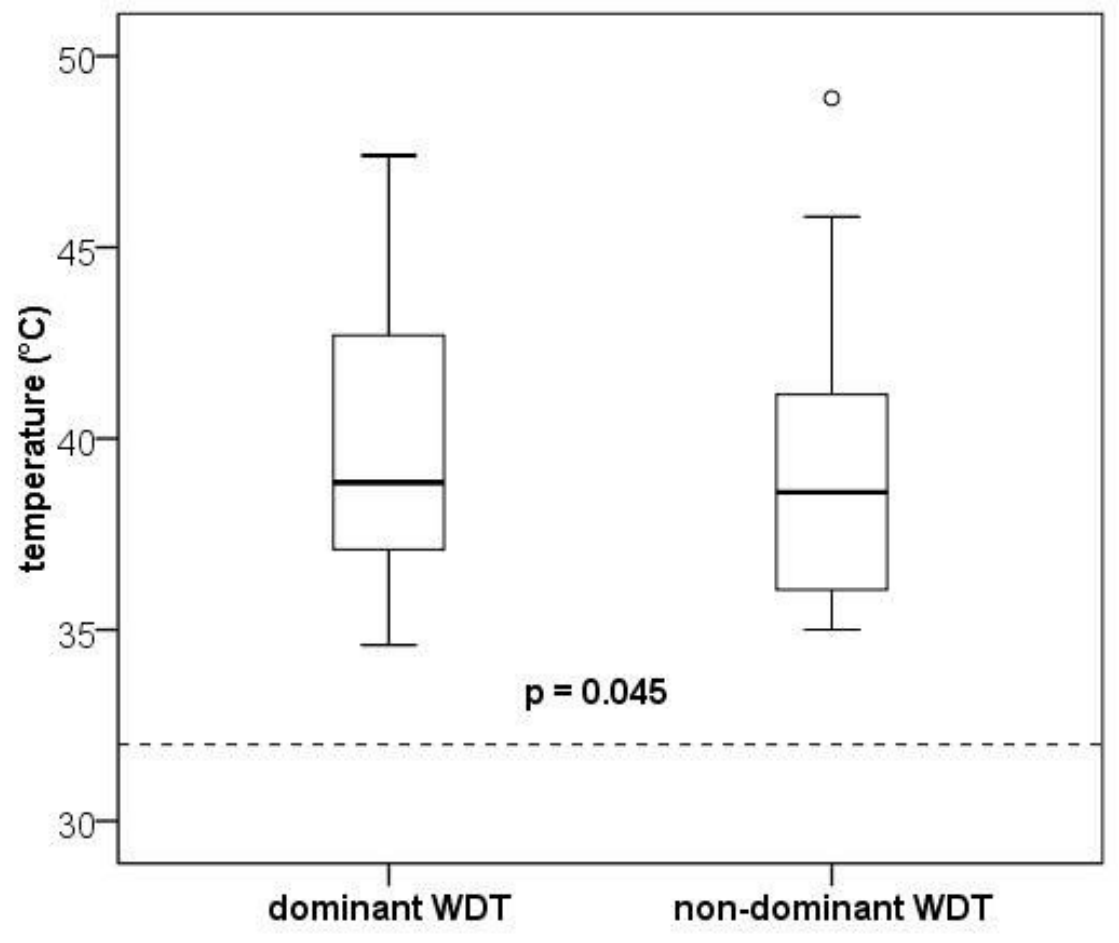

Figure 2

Warm detection threshold in Parkinson's disease patients. WDT, warm detection threshold; dominant, more affected side; nondominant, less affected side 